\title{
Generation of fluorescent silver nanoclusters in reverse micelles using gamma irradiation: low vs. high dosages and spectral evolution with time
}

\author{
Brett D. Martin $\cdot$ Jake Fontana $\cdot$ Zheng Wang $\cdot$ \\ Scott A. Trammell
}

Received: 1 April 2014/Accepted: 17 June 2014/Published online: 5 July 2014

(C) The Author(s) 2014. This article is published with open access at Springerlink.com

\begin{abstract}
Reverse micelles (RMs) containing aqueous solutions of $\mathrm{Ag}^{+}$ions in their core produce fluorescent $\mathrm{Ag}$ nanoclusters (NCs), upon exposure to gamma irradiation. The fluorescence spectra of the NCs evolve over days to weeks after the exposure, and usually show large increases in intensity. Responses of as high as $2.8 \times 10^{4} \mathrm{CPS} / \mathrm{Gy}$ were reached. A dosage as low as $0.5 \mathrm{~Gy}(10 \%$ of the lethal dosage for humans) produces NCs having fluorescence intensities higher than background. The RMs can be employed in novel gamma radiation detectors with appearance of fluorescence indicating that radiation was once present. In applications involving detection and tracking of fissile materials, the evolution of the fluorescence spectra over time may provide additional information about the radiation source. A two-phase liquid system is used for RM formation in a simple procedure. It is likely that this synthesis method may be adapted to produce NCs from other metal ions.
\end{abstract}

Keywords Reverse micelles - Silver nanoparticles · Silver nanoclusters · Gamma irradiation · Fluorescence . Fissile materials

\section{Introduction}

Metal nanoclusters (NCs) are at a length scale between atoms and nanoparticles and often have desirable

B. D. Martin $(\bowtie) \cdot$ J. Fontana $\cdot$ Z. Wang $\cdot$ S. A. Trammell

Naval Research Laboratory, Code 6900, Washington,

DC 20375, USA

e-mail: bdm@cbmse.nrl.navy.mil; brett.martin@nrl.navy.mil

S. A. Trammell

e-mail: scott.trammell@nrl.navy.mil properties typical of large organic molecules (bright fluorescence, for example) since their band structure is broken down into discrete energy levels ( $\mathrm{Xu}$ and Suslick 2010; Diez and Ras 2011). NC synthesis can be challenging, however, because they tend to aggregate in solution to form larger particles. Thus, molecular stabilizers and "nanoscaffolds" are used to prevent this. The reduction of $\mathrm{Ag}$ ions in these systems is carried out by chemical, photochemical or by radiolytic means (Zhang et al. 2005). In fact, both photochemical reduction and radiolysis have advantages since it is not necessary to introduce chemical reductants and perform subsequent purification (Belloni et al. 1998; Diez and Ras 2011; Zhang et al. 2012). Radiolytic reduction methods that produce Ag NCs in bulk aqueous solutions are straightforward and reproducible (Belloni et al. 1998). However, it appears that the generation of fluorescent NCs via this means is not common.

In a recent communication (Martin et al. 2012), we described our work using silver ion-containing reverse micelles (RMs) to generate fluorescent silver NCs upon exposure to gamma irradiation. In this system, the $\mathrm{Ag}^{+}$is sequestered into the aqueous core of the RMs. Gamma-ray exposure causes the water molecules contained in the core to undergo bond scission reactions, yielding solvated electrons, hydrogen atoms, and hydroxyl radicals (Woods and Pikaev 1994). This leads to rapid reduction of the $\mathrm{Ag}^{+}$ ions to $\mathrm{Ag}^{0}$ atoms which then aggregate into $\mathrm{NCs}$ that are often fluorescent. NC encapsulation in the RM prevents their further aggregation that would likely lead to loss of fluorescence, Eq. (1).

$$
\begin{gathered}
\text { gamma radiation }+\mathrm{H}_{2} \mathrm{O} \rightarrow \mathrm{e}_{\mathrm{aq}}^{-}+\cdot \mathrm{H}+\cdot \mathrm{OH}+\mathrm{H}^{+} \\
\stackrel{\mathrm{nM}+\text { reduction }}{\longrightarrow} n \mathrm{M}^{0} \stackrel{\text { aggregation }}{\longrightarrow} \mathrm{M}_{n}^{0} \text { (fluorescent) }
\end{gathered}
$$


We are interested in exploring the use of the RMs as detectors of gamma radiation emitted by fissile materials within the context of detection and tracking of radioactive isotopes used in weapons of mass destruction (WMD). For applications in detection and tracking of WMD based on radioactive fissile isotopes, we envision that the RMs could be suspended in a transparent resin (such as varnish) that would then be applied to the insides of shipping containers, for example. Periodic testing for fluorescence will reveal if gamma radiation was present at sometime in the past. In addition, the intensity and spectral characteristics of the fluorescence, as well as the manner in which they evolve over time (hours to weeks after exposure) may allow the exact time of the exposure to be calculated. In this paper, we investigate how the RM emission spectra vary as a function of dosage (very low vs. high), and the manner in which the spectra evolve over extended time periods.

\section{Experimental section}

\section{Materials}

Reagents were obtained as follows and used with no further purification: 3-( $\mathrm{N}$-morpholino) propanesulfonic acid (MOPS), $\mathrm{AgNO}_{3}$, and spectroscopic grade toluene were purchased from Sigma-Aldrich (St Louis, MO). The block copolymers (PS-PAA) were purchased from Polymer Source Inc. (Quebec, Canada).

\section{Formation of RMs}

In a typical two-phase synthesis of RMs containing an aqueous core, commercially available copolymer having a polystyrene block and a polyacrylic acid block (PS-PAA) was dissolved at a concentration of $2 \mathrm{mg} / \mathrm{mL}$ in $10 \mathrm{~mL}$ toluene. The solution was then placed into a $20-\mathrm{ml}$ vial. A 10-mM morpholine sulfonic acid (MOPS) buffer ( $\mathrm{pH} 7.2$ ) was prepared and silver nitrate was added in the dark to form a $10 \mathrm{mM}$ solution. Next, $10 \mathrm{~mL}$ of this aqueous solution was added to the toluene solution, a stir bar was placed in the vial; the vial was shielded from light and stirred at 30 RPM for either 16 or $72 \mathrm{~h}$ at room temperature. Afterwards, the stirring was stopped and $1 \mathrm{~mL}$ aliquots were then removed from the toluene phase (top phase). The aliquots were aerated (they were not sparged to remove dissolved $\mathrm{O}_{2}$ ).

\section{Radiation}

Most of the aliquots were subjected to gamma irradiation from a ${ }^{60} \mathrm{Co}$ source for various time durations, and the total doses were recorded. Other aliquots were not irradiated and used for control experiments. Irradiation studies were performed using a ${ }^{60} \mathrm{Co}$ source at the Naval Research Laboratory (Washington, DC). Samples were irradiated at dosage rates ranging from 0.5 to $508 \mathrm{~Gy} / \mathrm{min}$. Samples were stored in the dark after irradiation. Experiments were performed in triplicate.

\section{Characterization}

The set of aliquots was characterized using UV-visible and fluorescence spectrometry, and TEM imagery and analysis. UV-visible measurements were made using an Agilent 8453 diode array spectrometer and referenced against a solvent blank. Fluorescence measurements were made using a Fluoromax-3 (Horiba, Inc., Kyoto) fluorimeter, using a bandpass of $5 \mathrm{~nm}$ for the excitation and emission monochromators with integration set at $0.1 \mathrm{~s}$. Emission spectra were corrected using factors supplied by the manufacturer. For spectroscopy, 1-mL fluorescence quartz cuvettes were utilized. TEM imagery was performed using a JEOL (Tokyo) JEM-2200FS field emission electron microscope. An SPI 200 holey carbon mesh was coated on a $\mathrm{Cu}$ TEM grid, which was placed onto a Teflon sheet in a fume hood. Three $5-\mu \mathrm{L}$ droplets of toluene containing the Ag NCs were placed onto the grid at $1 \mathrm{~min}$ intervals, allowing for the toluene to evaporate between each drop. Also as reported previously (Martin et al. 2012), thermogravimetric analysis (TGA) and voltammetric stripping were performed using a Thermogravimetric Analyzer (Model 2950, TA Instruments, New Castle, DE), and a $\mathrm{CH}$ Instruments (Austin, TX) Model 760D potentiostat, respectively.

\section{Results and discussion}

In Fig. 1, we show our strategy for $\mathrm{Ag} \mathrm{NC}$ generation that uses RMs formed from block copolymers to confine the reactants in an aqueous core (Martin et al. 2012). The copolymers used are of relatively low MW and have only two blocks, one composed of polyacrylic acid (PAA) and the second composed of polystyrene (PS). Block copolymers such as this display useful solution and associative properties when they are dissolved in solvents that can dissolve one block, but not the other. One such associative structure is the RM (Riess 2003). Advantages of the PSPAA RM system include low critical micelle concentrations (Moffitt et al. 1996), a characterized water-solvent solubility equilibria (Gao et al. 1992), and some understanding of the scaling relationships between the size of the ionic blocks and metal ions for the formation of metal 


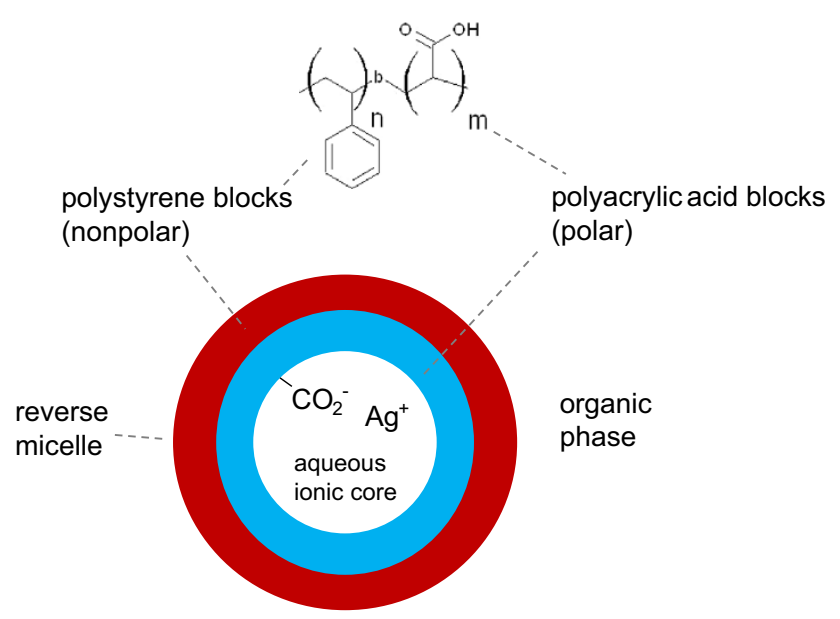

Fig. 1 PS-PAA block copolymer structure, and depiction of RM with aqueous ionic core having PAA carboxylate termini- $\mathrm{Ag}^{+}$ion pairs

nanoparticles (Moffitt et al. 1995; Bronstein et al. 1999; Hamley 2003; Wang and Moffitt 2004).

The aqueous core contains the $\mathrm{Ag}^{+}$and the AA carboxylate residues which may be largely ion paired since the morpholine sulfonic acid (MOPS) buffer maintains the $\mathrm{pH}$ at 7.2, higher than the $\mathrm{pK}_{\mathrm{a}}$ of the acrylic acid residues. When gamma radiation interacts with the aqueous ioncontaining core, solvated electrons and radical species (such as hydroxyl radicals) are created (Woods and Pikaev 1994). Here, the most likely reductant for the $\mathrm{Ag}^{+}$is the solvated electron. When aqueous solutions are irradiated at neutral and basic $\mathrm{pH}$, the solvated electron is the main reducing species (Woods and Pikaev 1994). The solvated electron then produces $\mathrm{Ag}^{0}$ from $\mathrm{Ag}^{+}$and the atoms associate to form Ag NCs.

In our initial communication (Martin et al. 2012), we used commercially available PS-PAA copolymers dissolved in toluene. Encapsulation of $\mathrm{Ag}^{+}$ions into the aqueous core was done using a two-phase liquid system with the polymer-toluene solution on the top, and the buffer on the bottom ( $\mathrm{pH} 7.2,10 \mathrm{mM}$ MOPS) containing $\mathrm{AgNO}_{3}$. The two phases were allowed to remain in contact with gentle stirring for $16 \mathrm{~h}$. The fluorescent Ag NCs were formed by exposing $1 \mathrm{~mL}$ aliquots of the toluene layer to gamma irradiation in a ${ }^{60} \mathrm{Co}$ pool at various doses. The toluene solutions that had not been irradiated showed no peaks in the visible absorbance spectra. Samples were exposed to the irradiation in a dosage range between 200 and 1,000 Gy. UV-visible spectra of the sample showed a broad visible absorption peak centered at $565 \mathrm{~nm}$, which we presumed arose from the Ag NCs. The results were consistent with literature reviews reporting that photoreductive methods with a PAA terpolymer template gave metal NCs with an absorbance centered at $\sim 500 \mathrm{~nm}$

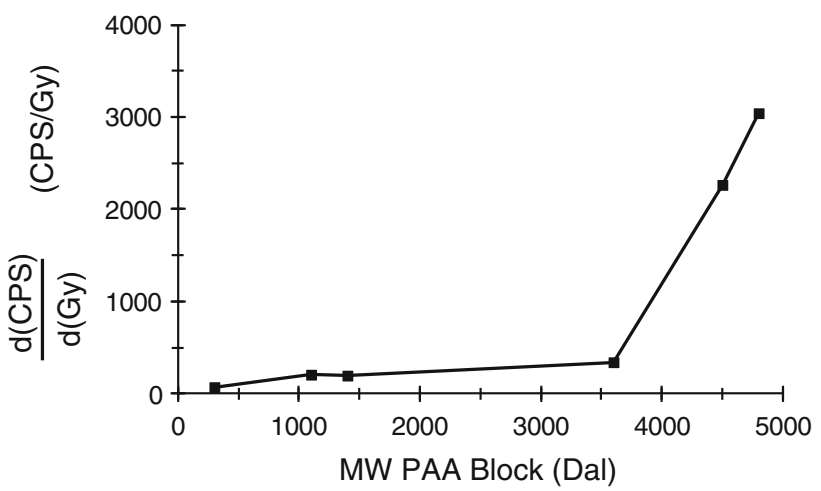

Fig. 2 For the set of copolymers examined, d(CPS)/d(Gy) plotted as a function of the PAA coblock MW. Error $\pm 6 \%$

Table 1 Block copolymers and dose responses

\begin{tabular}{lrrrr}
\hline Polymer \# & PS, MW & PAA, MW & Total MW & $\frac{\mathrm{d}(\mathrm{CPS})}{\mathrm{d}(\mathrm{Gy})}$ \\
\hline 1 & 11,000 & 300 & 11,300 & 71 \\
2 & 12,000 & 1,100 & 13,100 & 206 \\
3 & 19,000 & 1,400 & 20,400 & 196 \\
4 & 15,000 & 3,600 & 18,600 & 341 \\
5 & 42,000 & 4,500 & 46,500 & 2,264 \\
6 & 5,000 & 4,800 & 9,800 & 3,042 \\
\hline
\end{tabular}

$\mathrm{d}(\mathrm{CPS}) / \mathrm{d}(\mathrm{Gy})=$ change in fluorescence intensity in counts per second/change in dosage in Gy. Excitation $=565 \mathrm{~nm}$

(Belloni et al. 1998). Excitation at $565 \mathrm{~nm}$ generated a fluorescence spectrum with a broad emission maximum near $690 \mathrm{~nm}$, having an intensity that scaled linearly with dosage in the range 200-1,000 Gy. At the 1,000 Gy dosage, the response was 3,990 CPS/Gy where CPS denotes fluorescence photons (counts) per second. From the samples formed using a dose of 1,000 Gy, we estimated the fluorescent quantum yield of the Ag NCs to be $\sim 4.7 \%$. Control experiments with samples that were not irradiated, or solutions having no $\mathrm{Ag}^{+}$that were irradiated showed negligible fluorescence emission between 600 and $850 \mathrm{~nm}$.

To continue the investigation, we first wished to determine how the type of block copolymer used in RM formation ultimately affects the NC fluorescence. To do so, we used a set of commercially available PS-PAA block copolymers. In this study, the masses of copolymer and $\mathrm{AgNO}_{3}$ were held constant. For each polymer examined, a plot of fluorescence intensity vs. dosage was constructed for the dosage range 200-1,000 Gys. The plots were very closely linear and the slopes $\mathrm{d}(\mathrm{CPS}) / \mathrm{d}(\mathrm{Gy})$ were recorded. The slopes were plotted against the MW of the PAA coblock to generate Fig. 2, which shows that the slope increases significantly at a MW threshold between 3,600 and 4,800. Table 1 lists the various polymers used for RM formation, along with the corresponding value of $\mathrm{d}(\mathrm{CPS}) /$ 

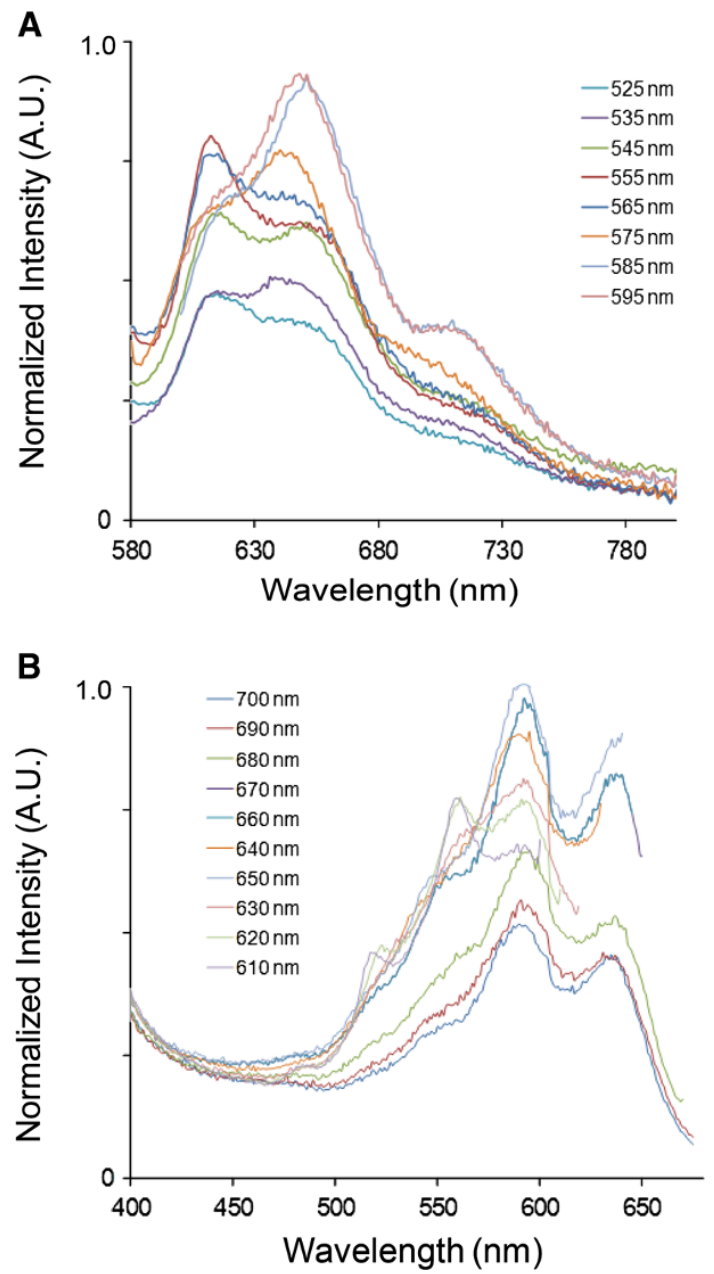

Fig. 3 Fluorescence emission (a) and excitation (b) spectra at 14 days after exposure to 5 Gys. In a, the legend denotes excitation wavelength; in $\mathbf{b}$, the legend gives the emission wavelength monitored

$\mathrm{d}(\mathrm{Gy})$ for each (excitation $=565 \mathrm{~nm})$. Polymer six has the largest value, with its roughly equal amounts of PS and PAA possibly enabling it to form especially stable RMs. Alternatively, the large number of PAA residues may simply allow sequestration of relatively more $\mathrm{Ag}^{+}$ions, thus a large number of $\mathrm{NCs}$ are formed, leading to the brighter fluorescence.

We then performed a fluorescence study in which we determined the manner in which the emission varied as a function of excitation wavelength through the range 525-595 nm, using low and high dosages. For the low dosages (5 Gys), the emission was measured by scanning through the range $610-700 \mathrm{~nm}$. The resulting fluorescence spectra are shown in Fig. 3a, with the chosen excitation wavelength values tabulated in the legend. We then performed the analogous study wherein the monitored emission wavelength was chosen to be at various points in the range $610-700 \mathrm{~nm}$, while the excitation wavelength was
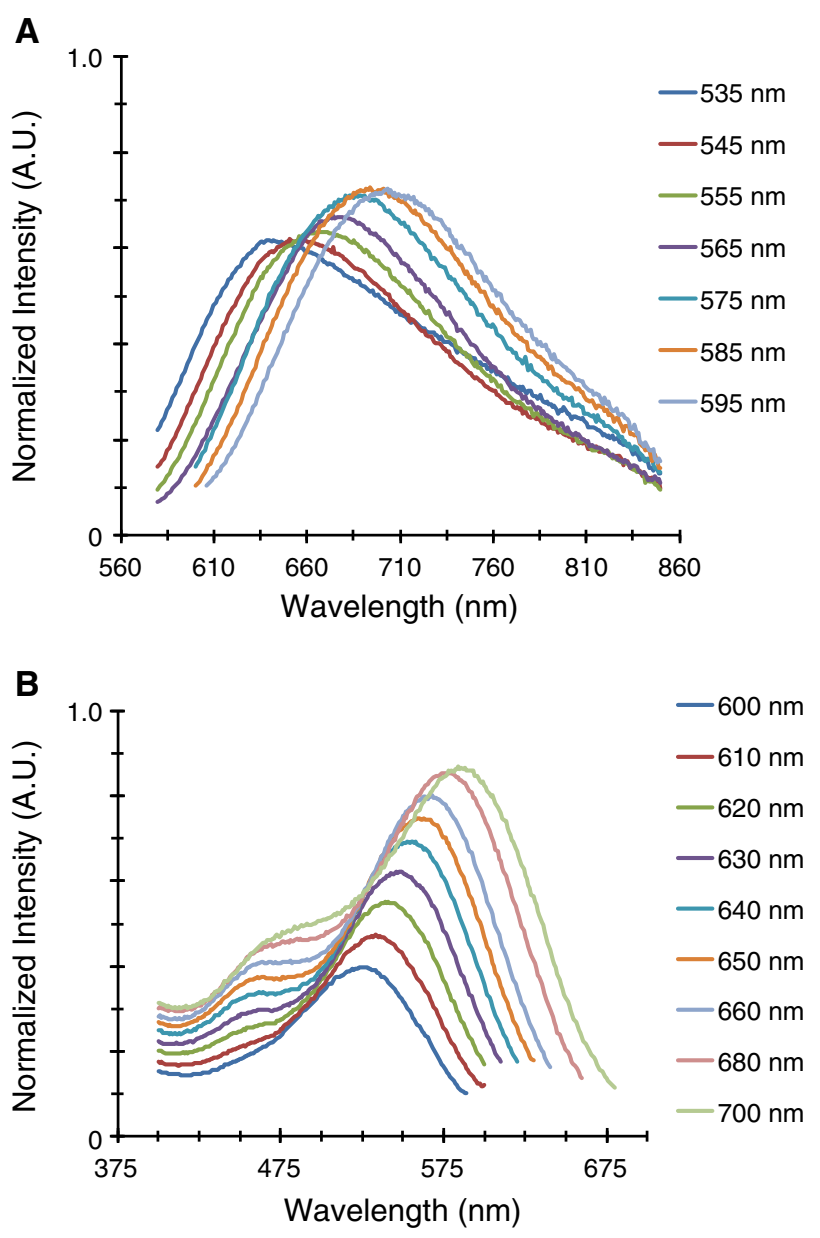

Fig. 4 Fluorescence emission (a) and excitation (b) spectra at $3 \mathrm{~h}$ after exposure to 1,000 Gys. In a, the legend denotes excitation wavelength; in $\mathbf{b}$, the legend gives the emission wavelength monitored

varied continuously through the range $400-670 \mathrm{~nm}$ (Fig. 3b). The spectra from samples that were not exposed to radiation (background spectra) have been subtracted from each. In Fig. 3a, the spectra have a considerable amount of structure, i.e., they all have three relative maxima at 611-613, 640-648, and 705-709 nm. As the excitation wavelength is increased from 525 to $595 \mathrm{~nm}$, the fluorescence intensity rises, then falls, and then rises again. In Fig. 3b, the spectra show even more structure, with all showing four relative maxima occurring at 517-522, $558-559,591-595$, and $630-636 \mathrm{~nm}$.

The spectra from the high (1,000 Gy) exposure are seen in Fig. $4 a, b$. Figure $4 a$ gives the emission spectra that resulted when the excitation frequency was varied. It is qualitatively similar to Fig. 3a (5 Gy dose), except that the spectra are much smoother (unstructured) with only one relative maximum. We then performed the analogous study wherein the excitation wavelength was varied continuously 
over the range $400-675 \mathrm{~nm}$, with the emission being monitored at points in the range $600-700 \mathrm{~nm}$ (Fig. 4b). Again, the spectra are qualitatively similar to the $5 \mathrm{~Gy}$ dose, but are much smoother with only one emission maximum. For the samples exposed to 1,000 Gy, the excitation and emission spectra show a wavelength dependence that is typical of systems that display inhomogeneous broadening and have recently been reported for $\mathrm{Ag}$ NCs generated photochemically in DMF (Diez et al. 2013). The lack of multiple relative maxima and associated structure may indicate that there is a much broader NC size distribution, with no set of discrete NC sizes. In an earlier study, we found that NCs formed using 1,000 Gy dosages were relatively large ( $\sim 20 \mathrm{~nm}$ diameter $)$ and were thought to contain "islands" of smaller fluorescent clusters in a non-fluorescent matrix (Martin et al. 2012).

Next, we wished to determine (1) what is the mass composition of the RMs immediately after formation (in terms of the amounts of $\mathrm{Ag}^{+}$and block copolymer present)?, and (2) how does the consumption rate of the $\mathrm{Ag}^{+}$ depend on dosage level?

In our initial communication, the amounts of polymer and $\mathrm{Ag}^{+}$were found using thermogravimetric analysis (TGA) and anodic stripping square wave voltammetry (ASSWV). In the toluene phase, we found that the $\mathrm{Ag}^{+}$and the polymer were present at levels of 0.57 and $0.057 \mathrm{mM}$, respectively (Martin et al. 2012). Thus, the molar concentration of the $\mathrm{Ag}^{+}$was tenfold higher than that of the polymer. This implied that the PAA portion of each block copolymer molecule in the RM was ion paired with $10 \mathrm{Ag}^{+}$ ions. Using both irradiated and non-irradiated samples we extracted the $\mathrm{Ag}^{+}$ions from the RM-toluene solution into buffer. We then performed ASSWV and calculated the amount of $\mathrm{Ag}^{+}$that had reacted (Martin et al. 2012). Interestingly, we found that the highest $\mathrm{Ag}^{+}$consumption rates occurred at the lowest dose of 50 Gys. However, only relatively weak fluorescence appears at $50 \mathrm{~Gy}$. Evidently, at that dosage a large population of Ag NCs is formed, with (1) all having weak fluorescence that may change over time, or (2) some having weak fluorescence that may change over time, but others being pre-fluorescent or permanently non-fluorescent. A key question thus emergedhow does the spectral evolution of the weakly fluorescent and pre-fluorescent species occur over time?

This gave us added incentive to explore how low gamma doses, combined with post-exposure time-dependent spectral analysis, and ways of treating the RMs post-exposure could ensure maximum, or the most efficient, transformation of the weakly- and pre-fluorescent species into bright fluorescent ones. Our goal was to use low doses of $0.5-5$ Gy to achieve fluorescence intensities equal to those attained from exposure to several $100 \mathrm{~Gy}$ or higher in our previous work. As mentioned in the "Introduction", this

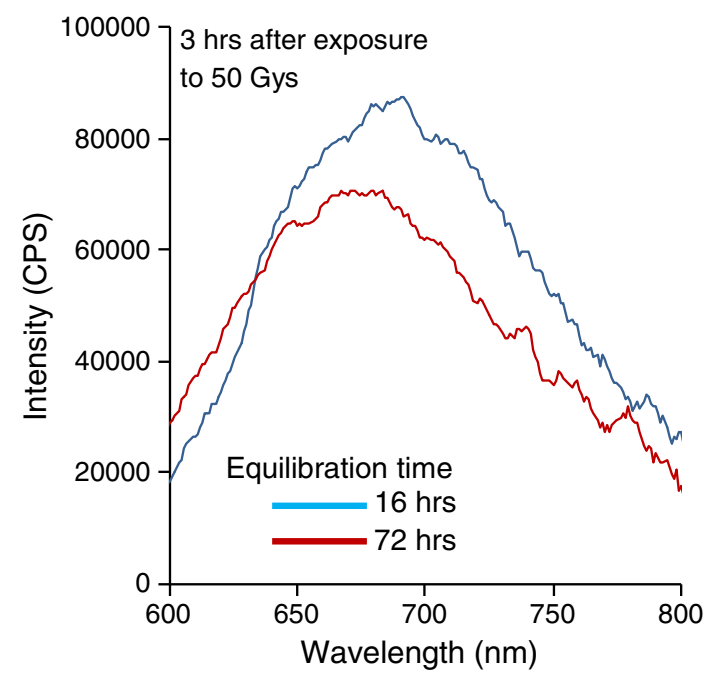

Fig. 5 NC emission intensity as a function of the 2-phase equilibration times. Excitation $=565 \mathrm{~nm}$

may ultimately allow development of gamma radiation detectors with much higher sensitivities, and allow development of synthesis methods for fluorescent NCs that will require much lower irradiation energies.

Before we proceeded with the low-dosage studies, we decided to investigate whether the RM formation process and ultimately NC fluorescence could be affected using a longer equilibration time for the two liquid phases (toluene and buffer). Dosages of 2 and 50 Gy were examined, using an excitation wavelength of $565 \mathrm{~nm}$. For the 2 Gy dosage, the 72-h equilibration time led to a consistently higher fluorescence intensity (1,080 CPS at $680 \mathrm{~nm}$, for example) than the $16 \mathrm{~h}$ time, which yielded levels very near zero (the background spectra were subtracted), but no clear relative maxima appeared in these spectra. In contrast, for the 50 Gy dosage, the 16-h equilibration time was optimal, leading to a clear relative maximum having a peak intensity that was $\sim 20 \%$ higher than that attained from the $72 \mathrm{~h}$ time (Fig. 5). Thus, the longer equilibration time leads to higher fluorescence intensities for the 2 Gy dosage (but with no relative maxima), and the shorter equilibration time leads to higher intensities for the 50 Gy dosage (with a relative maxima present). The mass transfer that occurs across the liquid-liquid phase boundary is probably a dynamic process involving time-dependent formation of RM populations having variable sizes and contents. The longer equilibration times may allow the polymer to enter further self-assembly processes that lead to RMs responsive to the lowest dosages, but not to the higher dosages. The relationship between RM formation, dosage, and NC development is an attractive area for further investigation. Another important area of study is the effect of polymer MW and composition on the polymer solubility in each liquid phase. 

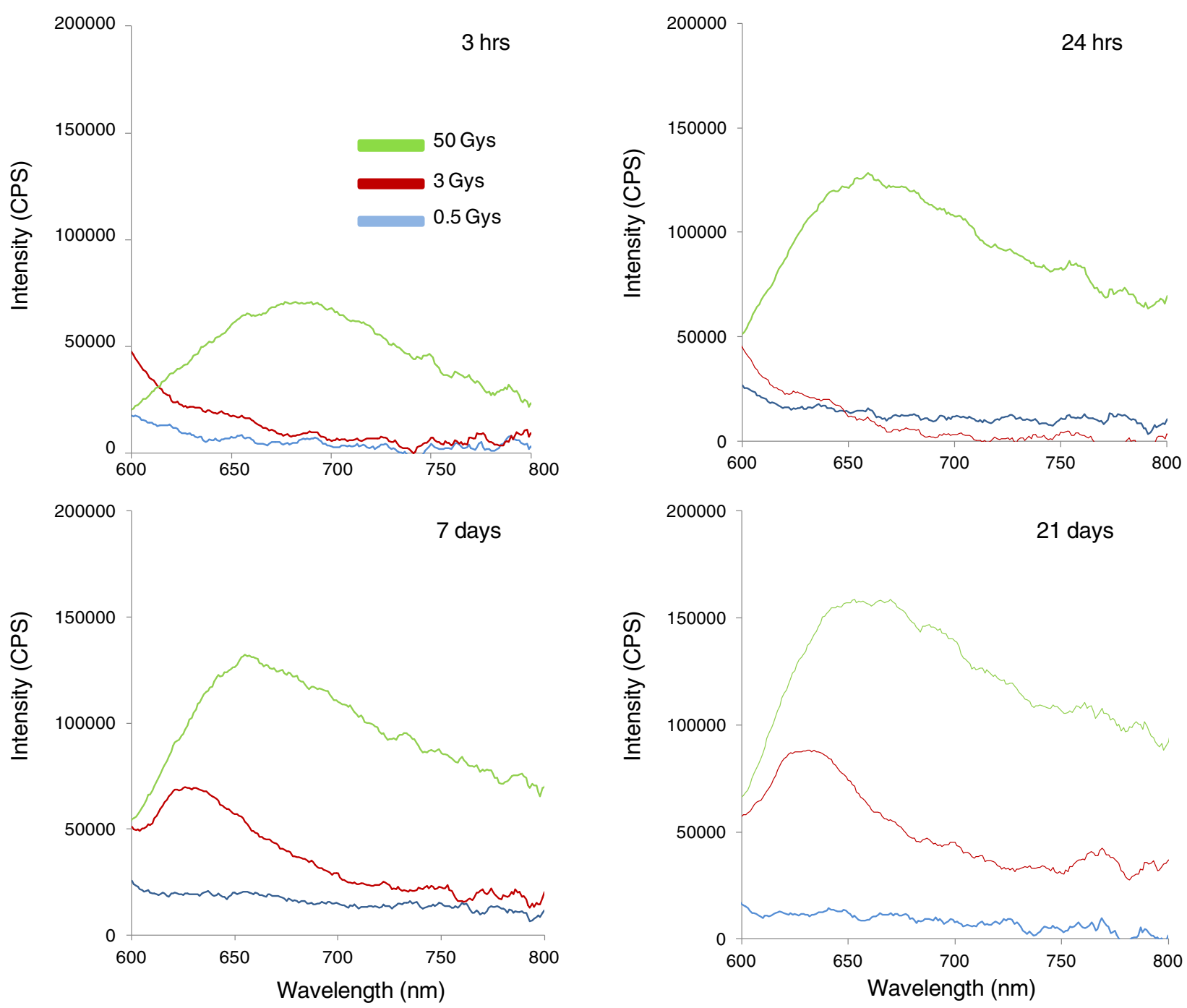

Fig. 6 Dosage and time-dependent spectral evolution of the NCs, from $3 \mathrm{~h}$ to 21 days after exposure

For the next set of studies using the lower dosage range 0.5-50 Gy, the RMs were formed using the standard twophase system, with the 72-h equilibration time. The long equilibration time was chosen to maximize detection sensitivity in the lowest dosage range. The samples were exposed to gamma irradiation in the same manner as before. Their resulting visible absorption spectra showed very low absorptivities, and are not shown. However, the $\mathrm{NC}$ fluorescence spectra showed significant dose-dependent differences (excitation maximum at $565 \mathrm{~nm}$ ) and changed gradually over hours and days after exposure (Fig. 6). The spectra from samples that were not exposed to radiation (background spectra) have been subtracted from each. Several phenomena are apparent from the time course. First, the relatively very low dose of 0.5 Gys is sufficient to cause formation of fluorescent NCs. However, they do not have a well-defined emission maximum, and there is no significant spectral change over time. For the higher dose of $3 \mathrm{~Gy}$, the early spectra are similar to those for the 0.5 Gy exposure over the first few days. However, at $t=7$ days an emission maximum at $630 \mathrm{~nm}$ appears. This may indicate that the NC size distribution may have narrowed to favor a certain size range. Over longer times up to 21 days, the fluorescence intensity increases slightly. For the highest dose of $50 \mathrm{Gys}$, the emission maximum at $680 \mathrm{~nm}$ appears at $3 \mathrm{~h}$ after exposure. Over the 21-day time course the fluorescence intensity increases, and the position of the emission maximum becomes blue shifted to $655 \mathrm{~nm}$. For the spectra having a definite emission maxima (Fig. 6), the largest response is $2.8 \times 10^{4} \mathrm{CPS} / \mathrm{Gy}$, from the $3 \mathrm{~Gy}$ dose. Figure 7 shows the time-dependent emission intensity at the wavelength of maximum emission. For the 3 and 50 Gy exposures, a dramatic increase in intensity appears at a distinct point in the time course with the increase for the higher dose occurring sooner. 


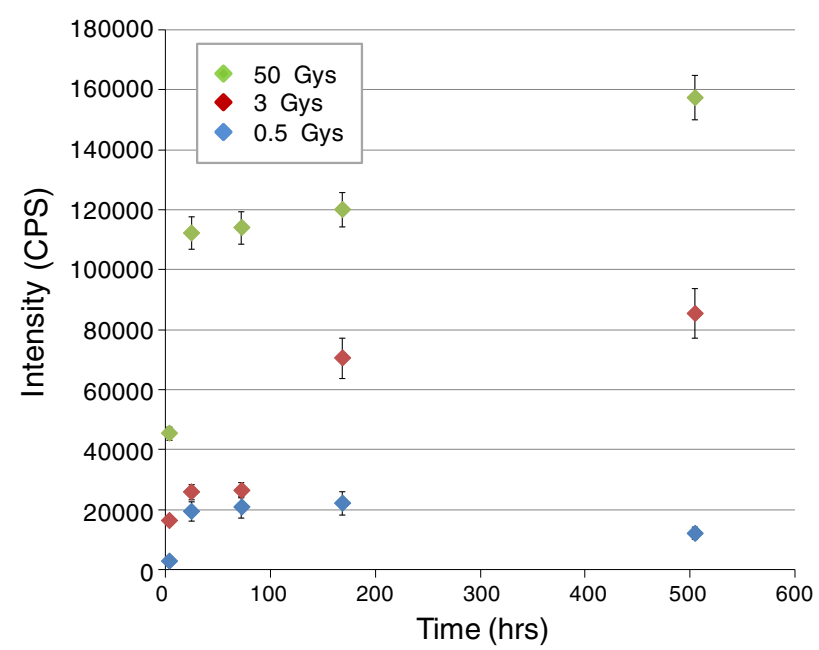

Fig. 7 Dosage and time-dependent intensity of emission at $\lambda_{\max }$, from $3 \mathrm{~h}$ to 21 days after exposure

Table 2 Results from low dosages

\begin{tabular}{|c|c|c|c|}
\hline Sample history & $\begin{array}{l}\text { Dose } \\
\text { (Gys) }\end{array}$ & $\begin{array}{l}\text { Response } \\
\text { (CPS/Gy) }\end{array}$ & Fluorescence spectra \\
\hline \multirow{2}{*}{$\begin{array}{l}\text { Tested at intervals over } \\
21 \text { days }^{\mathrm{a}}, 72-\mathrm{h} \mathrm{PET}^{\mathrm{b}}\end{array}$} & 3 & $2.8 \times 10^{4}$ & \multirow{2}{*}{$\begin{array}{l}\lambda_{\max } \text { at later } t(3 \mathrm{~Gy}) \\
\quad \text { broad } \lambda_{\max } \text {, all } \\
t(50 \mathrm{~Gy})\end{array}$} \\
\hline & 50 & $3.2 \times 10^{3}$ & \\
\hline
\end{tabular}

a After exposure

b Phase equilibration time

The data in Figs. 6 and 7 reveal that the NC formation processes are temporally dynamic. Properties such as average size and fluorescence intensity (ultimately quantum yield) are dependent on processes that are time dependent. The fluorescence intensity undergoes conspicuous step-like changes that are in conjunction with long time periods with little apparent change. Examples of processes that may underlie these changes are rates of silver atom aggregation/deaggregation, rates of cluster aggregation/deaggregation, and diffusion rates of atoms and clusters. Table 2 summarizes the data shown in Figs. 6 and 7.

Figure 8a, $\mathrm{b}$ shows TEM images of NCs formed at the various doses. They were imaged at 21 days after formation. For comparison, NCs that formed spontaneously without exposure to irradiation are also shown. Those formed in this manner have negligible levels of fluorescence. The NCs that formed without exposure have a clear non-homogeneous size distribution ranging from 2 to 3 to $\sim 15 \mathrm{~nm}$. In contrast, those formed via gamma-ray exposure have highly uniform size distributions, in the range $8-10 \mathrm{~nm}$. It is of interest that there is virtually no variation in NC size as a function of dosage in the range $0.5-50 \mathrm{~Gy}$. However, comparison at higher magnification (Fig. 8b)
A

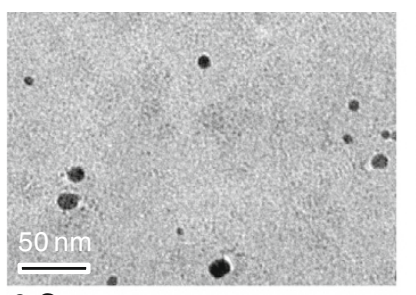

0 Gys

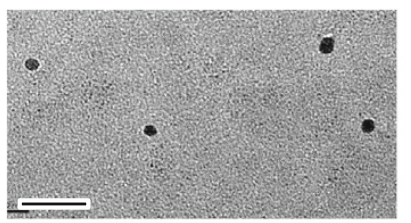

3 Gys

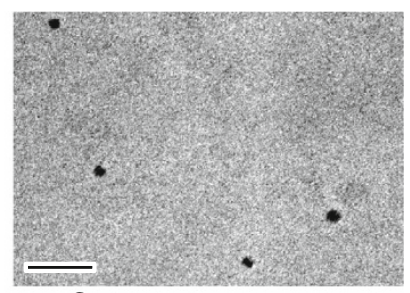

0.5 Gys

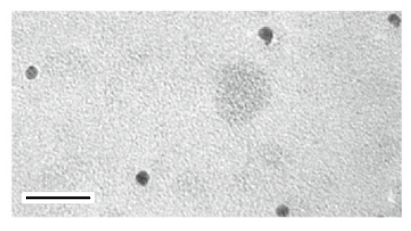

50 Gys

B

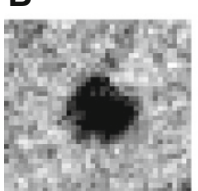

0.5 Gys

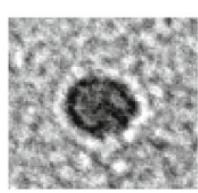

3 Gys
50 Gys

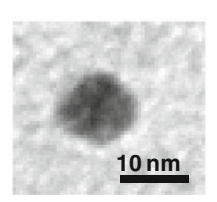

Fig. 8 a, b TEM images of Ag NCs formed at various gamma-ray dosages, and control (0 Gy)

shows that the 3 and the 50 Gy NCs are structurally more similar to one another than to the 0.5 Gy NCs. Finally, close examination of the NCs in Fig. 8b reveals that they appear to be a conglomerate of smaller NCs. This is especially apparent for the NCs formed using 3 and $50 \mathrm{~Gy}$.

It is also of interest that the background region appears to have a structure of its own, most readily seen in the images having a higher magnification. This structure was not visible when samples not having the block copolymer were imaged. The structure may in fact be the block copolymer adsorbed onto the silicon surface, made visible in TEM by residual $\mathrm{Ag}^{+}$that is ion paired to the PAA repeat units.

The findings depicted in Figs. 6, 7 and 8 can be summarized as follows. The NC size range attained via the 0.5 Gy dosage nearly matches the size range arising from the 3 and 50 Gy dosage, but in the latter two cases (1) the fluorescence intensity is $\sim 8$ and 16-fold higher, respectively, (2) a pronounced emission maxima becomes present, (3) the fluorescence intensity gradually increases over time, (4) a close structural similarity is seen, (5) they appear to be a conglomerate of smaller NCs. The NCs formed using the dose range 3-50 Gy are, evidently, profoundly different from those formed using a $0.5 \mathrm{~Gy}$ dosage. It may simply be that the low dosage does not generate enough solvated electrons through water scission, and the $\mathrm{Ag}^{+}$enters other chemical pathways that lead to formation of NCs with less desirable characteristics. 
TEM imagery showed that the NCs formed using 3 and $50 \mathrm{~Gy}$ appear to be a conglomerate of smaller NCs. Each relative maxima seen in both fluorescence plots may represent a particular NC size, or narrow size distribution, present in the conglomerate. Thus, four discrete NC sizes/ size distributions may be present.

\section{Conclusions}

Five main conclusions can be drawn from our work presented here, which can be summarized as follows:

1. In initial studies using the dosage range 200-1,000 Gys, fluorescence measurements $3 \mathrm{~h}$ after exposure show linear relations between emission intensity and dosage, with emission maxima occurring near $690 \mathrm{~nm}$. A 16-h phase equilibration time was used.

2. The block copolymer leading to NCs with the highest response (3,042 CPS/Gy @ 1,000 Gys) had roughly equal lengths of PS and PAA.

3. RM mass composition studies (1) suggest that the PAA portion of each block copolymer molecule in the RM is ion paired with $10 \mathrm{Ag}^{+}$ions, and (2) show that the highest $\mathrm{Ag}^{+}$consumption rate occurs at lower dosages.

4. The fluorescence intensity of $\mathrm{NCs}$ formed using dosages of $0.5-50$ Gys evolves over a 21-day time period. Dosages of 3 and 50 Gys result in intensities and emission maxima that generally increase over this time. The maxima undergo conspicuous step-like changes that are in conjunction with long time periods with little apparent change.

5. TEM imagery shows that dosages of 3 and 50 Gys lead to NCs having a very narrow size distribution, with diameters of $8-10 \mathrm{~nm}$. A close structural similarity is seen, and they appear to be a conglomerate of smaller NCs.

Acknowledgments This work was sponsored by the Defense Threat Reduction Agency. (MIPR \# 10-2650M, 11-2198M and HDTRA 121916)

Open Access This article is distributed under the terms of the Creative Commons Attribution License which permits any use, distribution, and reproduction in any medium, provided the original author(s) and the source are credited.

\section{References}

Belloni J, Mostafavi M, Remita H, Marignier JL, Delcourt MO (1998) Radiation-induced synthesis of mono- and multi-metallic clusters and nanocolloids. New J Chem 22(11):1239-1255

Bronstein LH, Sidorov SN, Valetsky PM, Hartmann J, Colfen H, Antonietti M (1999) Induced micellization by interaction of poly(2-vinylpyridine)-block-poly(ethylene oxide) with metal compounds. Micelle characteristics and metal nanoparticle formation. Langmuir 15(19):6256-6262

Diez I, Ras RHA (2011) Fluorescent silver nanoclusters. Nanoscale 3(5):1963-1970

Diez I, Ras RHA, Kanyuk MI, Demchenko AP (2013) On heterogeneity in fluorescent few-atom silver nanoclusters. Phys Chem Chem Phys 15(3):979-985

Gao ZS, Desjardins A, Eisenberg A (1992) Solubilization equilibria of water in nonaqueous solutions of block ionomer reverse micelles-an NMR-study. Macromolecules 25(4):1300-1303

Hamley IW (2003) Nanostructure fabrication using block copolymers. Nanotechnology 14(10):R39-R54

Martin BD, Fontana J, Wang Z, Louis-Jean J, Trammell SA (2012) Generation of fluorescent silver nanoscale particles in reverse micelles using gamma irradiation. Chem Commun 48(86): 10657-10659

Moffitt M, McMahon L, Pessel V, Eisenberg A (1995) Size control of nanoparticles in semiconductor-polymer composites. 2. Control via sizes of spherical ionic domains in styrene-based diblock ionomers. Chem Mater 7(6):1185-1192

Moffitt M, Khougaz K, Eisenberg A (1996) Micellization of ionic block copolymers. Acc Chem Res 29(2):95-102

Riess G (2003) Micellization of block copolymers. Prog Polym Sci 28(7):1107-1170

Wang CW, Moffitt MG (2004) Surface-tunable photoluminescence from block copolymer-stabilized cadmium sulfide quantum dots. Langmuir 20(26):11784-11796

Woods RJ, Pikaev AK (1994) Applied radiation chemistry: radiation processing. Wiley, USA

Xu HX, Suslick KS (2010) Water-soluble fluorescent silver nanoclusters. Adv Mater 22(10):1078-1082

Zhang JG, Xu SQ, Kumacheva E (2005) Photogeneration of fluorescent silver nanoclusters in polymer microgels. Adv Mater 17(19):2336

Zhang H, Huang X, Li L, Zhang G, Hussain I, Li Z, Tan B (2012) Photoreductive synthesis of water-soluble fluorescent metal nanoclusters. Chem Commun 48(4):567 